\title{
The Netherlands Advancing to the Forefront of Regenerative Medicine
}

\author{
Kurt Gielen*
}

ABSTRACT

The country, and especially Limburg, the most southern province, is making major leaps forward in the commercialization of regenerative medicine. Chemelot Campus, a major business park, is making a massive commitment with the creation of "Regeneration Street"; $50,000 \mathrm{~m}^{2}$ of world leading research, development, manufacturing and innovation in regenerative medicine.

\section{Knowledge is key}

The Netherlands is investing strategically in networks focused on open innovation and international collaboration. The long tradition of collaboration between companies, knowledge and educational institutes, and the governments is internationally recognized and admired. The reasons for doing this are numerous. The world is changing fast. We are faced with a shortage of raw materials, the population is aging rapidly, the climate is changing, and the need for food and energy is rising. The international competition is increasing, for one because of the extended growth in Asia.

That is why the federal government is investing strategically in top sectors, including life science and chemistry. Innovation especially is going to assure economic growth in these sectors.

Chemelot Campus B.V., Geleen, the Netherlands. *(Correspondence: kurt.gielen@brightlands .com)

\section{Become a top technology} region in the world

The goal for the southeast of the Netherlands is to become one of the world's top technology regions with sufficient employment for all inhabitants. The southeastern region of the Netherlands is already responsible for $35 \%$ of the country's export, $45 \%$ of all private investments in research and development, and $55 \%$ of all patent requests. This region is a breeding ground for innovation and home to world-class companies and to research and knowledge institutes. Together, they invent and develop the technologies of tomorrow that will contribute to a safer, greener, and more caring society and a sustainable economic development for the region and the country.

For Limburg, the most southern province of the Netherlands, this is an excellent opportunity due to its long history in innovation and its strategic location. Limburg is situated in the heart of the European region between two major rivers, the Meuse and the Rhine. An area where nearly 4 million people live across
At Chemelot Campus in Limburg, there are an average of 250 patents and dozens of new companies every year.

three countries: The Netherlands, Belgium, and Germany.

The key economic areas in Limburg are chemistry and materials, life sciences and health, and smart services. Chemelot Campus and Maastricht Health Campus act as the two most important growth engines of the region. They contribute to an excellent business climate and are in this way structurally reinforcing the economy of the province of Limburg.

\section{Biomedical materials,}

\section{tissue engineering, and regenerative medicine}

Biomedical materials, tissue engineering, and regenerative medicine are some of the most promising fields of innovation where the Netherlands is making significant leaps forward. Chemelot Campus and Maastricht Health Campus play a key role in these advancements. On an academic level, this is driven by the University and Academic Hospital of Maastricht, together with the Clinical Trial Center Maastricht, their unique professional 
partner for all clinical trials and other human-related research.

The campuses are home to fast-growing startups such as CytuVax (cancer vaccines platform), Amarna Stem Cells (development and exploitation of an autologous bone marrow-derived stem cell platform), Xilloc Medical (additive manufacturing of patient-specific implants to close bone defects of the human skull), and more established companies like Tigenix (first company to receive approval for cell-based therapy in Europe) and PharmaCell (a global leader as Contract Manufacturing Organization [CMO] in regenerative medicine).

\section{Chemelot Campus: 100+ years of innovation}

Chemelot Campus is an open innovation campus in Sittard-Geleen. Chemelot Campus offers a vibrant community of innovative world-leading companies and knowledge institutes, state-of-the-art high-tech R\&D and manufacturing infrastructures, and venture capital and business development services to the companies on the campus and companies that establish a subsidiary. Over 50 companies currently present at Chemelot Campus are focused on R\&D in the fields of performance materials, biobased materials, and biomedical materials. Biomedical materials comprise regenerative medicine, tissue engineering, drug delivery systems, medical coatings, and implants. With its location at the axis of the cross-border region of the Netherlands-Belgium-Germany and the offices of the European Parliament, the campus is a European stepping stone for global expansion. This unique position attracts innovative companies, unique knowledge institutes, and visionary investors. Chemelot Campus is a junction where entrepreneurs and companies accelerate each other's growth and students create and find their new jobs. This leads to an average of 250 patents and dozens of new companies every year.
Chemelot Campus collaborates with local partners such as Maastricht Health Campus and international partners like RWTH Aachen and Technical University in Eindhoven. This has led to the establishment of two border-crossing research institutes, Chemelot InSciTe and the Aachen-Maastricht Institute for Biobased Materials.

\section{Chemelot and}

\section{regenerative medicine}

Regenerative medicine is a new dimension in the pharma world. Its goal is to invent, build, and supply innovative solutions by tapping into the healing powers of our own bodies through the use of autologous or allogeneic cells, in some cases combined with biomaterials that serve as scaffolds or delivery mechanisms for the human cells. The aim is to maintain healthcare at reasonable costs by moving from a palliative, disease-controlling healthcare system to solutions that actually cure the disease, apart from prevention. This development offers enormous market opportunities for regenerative medicine. However, the scientific, technological, and definitively the manufacturing developments are still in their infancy. Large progress can be

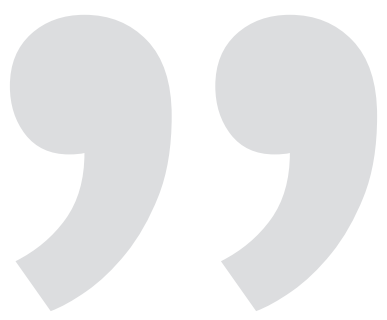

The aim is to maintain healthcare at reasonable costs by moving from a palliative, diseasecontrolling system to solutions that actually cure the disease.

\section{By the Numbers}

$\$ 900$ million: The estimated amount of the regenerative medicine industry in 2013.

2,500: The number of regenerative medicine clinical trials currently taking place. 500: Current regenerative medicine clinical trials in latestage development. $60 \%$ : The approximate success rate from phase III trials to market approval.

300: The number of innovative regenerative medicine products expected to enter the market, based on current trial figures.

made in terms of cost reduction and production efficiency especially because the product that is being manufactured is a living entity, often a patient-specific product. By definition, this product differs from patient to patient. These variations require a unique set of skills to define product specifications and to design manufacturing processes that deliver a consistent quality. The regenerative medicine industry is estimated to be a \$900-million industry in 2013.

The industry is currently at a tipping point and is positioned to hit its next phase of growth in the coming years. An important indicator for that is the number of clinical trials that are currently going on: 2,500 disruptive regenerative medicine-based clinical trials right now, of which over 500 are in late stage. With today's success rate of about $60 \%$ from phase III to market approval, this means we can expect over 300 highly innovative products to enter the market, and this brings with it an entirely new set of challenges.

Among others, these challenges concern optimizing and scaling up manufacturing processes, and expanding production capabilities and facilities. This is where Chemelot Campus comes in. With its unique expertise in high-tech and R\&D infrastructures, scalable production 
plants, and good manufacturing practices, related to innovative industries, Chemelot Campus is committed to play a defining role in the coming years. This commitment is stimulated by the fact that two of the three approved advanced therapy medical products (ATMPs) in Europe are produced in the (Dutch) province of Limburg, where Chemelot Campus is located. Chemelot Campus is accelerating the commercial manufacturing of living human cells for medical treatment, the production of living tissue, and (in the distant future) completely new organs. Globally, there are over 700 companies with patents and innovations in this field. Many of these will need dedicated manufacturing facilities with skilled and trained employees and the appropriate QC systems, preferably in a scalable and modular manner so that the facilities can grow and develop as the individual company develops.

\section{Regeneration Street}

In 2014, Chemelot Campus announced its first step in the realization of the so-called Regeneration Street on its premises, comprising $50,000 \mathrm{~m}^{2}$ of world-leading research, development, manufacturing, and innovation in regenerative medicine.

The first step is a fully equipped and serviced state-of-the-art building, simply called Regeneration Street nr. 1. This will be home to ATMP manufacturing companies, research institutes, a contract manufacturing organization, and startups in the fields of regenerative medicine and tissue engineering. It offers an advanced facility, open for all regenerative medicine companies, regardless of what stage of development they are in. Regeneration Street nr. 1 offers flexibility in setup and scalability through an innovative architecture of the interior. The building has a total ground floor size of $2,400 \mathrm{~m}^{2}$, with $750 \mathrm{~m}^{2}$ clean-room facilities. Regeneration Street nr. 1 is already up and running and capacity will be expanded by Q4 of this year.

\section{PharmaCell as contract}

\section{manufacturing organization}

PharmaCell plays an important role in the realization of Regeneration Street nr. 1. The company rents the facility, using it as the production facility for its larger scale CMO activities. Among other products, PharmaCell manufactures the Tigenix' ChondroCelect ${ }^{\circledR}$ product.

PharmaCell will also act as a service provider for the facility to ensure that required $\mathrm{QC}$ controls are met, which offers other partners of the facility a significant advantage in time and cost to get their manufacturing up and running according to the highest standards.

\section{New research institute}

A significant part of the facility will be rented to the Chemelot Institute for Science and Technology, Chemelot InSciTe. Chemelot InSciTe is a unique publicprivate collaboration of the Eindhoven University of Technology, Maastricht University/Medical Center, DSM, a global leader active in health, nutrition and materials, and Chemelot Campus. One of the goals of Chemelot InSciTe is to develop smarter biomedical materials to be able to sustain the ever more expensive healthcare. Chemelot InSciTe combines the forces, knowledge, and expertise of talented scientists, pioneering R\&D-focused companies, and innovative laboratories active in the field of biomedical materials.

The founding fathers have jointly invested $€ 40$ million for the coming 6 years and the total budget will be $€ 80$ million, thereby stimulating both employment and further development of knowledge and activity in the Limburg region and beyond. For biomedical applications, the institute will be working on, among other things, materials for new blood vessels, materials by means of which cartilage can repair itself and regenerate in the body, and clever miniature "containers" of medicines that are placed in the eye to prevent and treat eye infections.

Although this report includes some forward-looking statements and predictions made of clinical products on the market and the successful treatment of all kinds of diseases is indeed a prediction, it is obvious that this innovative field of healthcare and medicine is making swift progress. The south of the Netherlands is proud to be a global leader and an active participant in making this bright future become reality.

\section{Author Disclosure}

\section{Statement}

No competing financial interests exist. 